\title{
A Hierarchical Control Algorithm for Managing Electrical Energy Storage Systems in Homes Equipped with PV Power Generation
}

\author{
Yanzhi Wang, Siyu Yue, and Massoud Pedram \\ Department of Electrical Engineering \\ University of Southern California \\ Los Angeles, CA, USA \\ \{yanzhiwa, siyuyue,pedram\}@usc.edu
}

\author{
Louis Kerofsky and Sachin Deshpande \\ Sharp Laboratories of America \\ Camas, WA, USA \\ \{lkerofsky, sdeshpande $\} @$ sharplabs.com
}

\begin{abstract}
Integrating residential-level photovoltaic ( $\mathrm{PV})$ power generation and energy storage systems into the smart grid will provide a better way of utilizing renewable power. This has become a particularly interesting problem with the availability of dynamic energy pricing models in which electricity consumers can use their PV-based generation and controllable storage devices for peak shaving on their power demand profile from the grid, and thereby, minimize their electric bill cost. The residential-level storage controller should possess the ability of forecasting future PV-based power generation and load power consumption profiles for better performance. In this paper we present novel PV power generation and load power consumption prediction algorithms, which are specifically designed for a residential storage controller. Furthermore, to perform effective storage control based on these predictions, we separate the proposed storage control algorithm into two tiers, one which is performed at decision epochs of a billing period (e.g., a month) to globally "plan" the future discharging/charging schemes of the storage system, and another one performed locally and more frequently as system operates to compensate prediction errors. The first tier of algorithm is formulated and solved as a convex optimization problem at each decision epoch of the billing period, while the second tier has $O(1)$ complexity.
\end{abstract}

Keywords-PV; storage; prediction; hierarchical control

\section{INTRODUCTION}

The traditional static and centralized structure of electricity grid consists of a transmission network, which transmits electrical power generated at remote power plants through long-distance high-voltage lines to substations, and a distribution network, which delivers electrical power from substations to local end users. Since the end user profiles often change drastically according to the day of week and time of day, the Power Grid must be able to support the worst-case demand of power to all end users [1].

To avoid expending a large amount of capital for expanding the power generation capacity to meet the expected growth of end user energy consumption at the worst case, integration of smart meters aims to transform the Power Grid to a decentralized Smart Grid, which can monitor and control the power flow in the Grid to match the amount of power generation to that of the power consumption, and to minimize the overall cost of electrical power delivered to the end users [2]. Utility companies can deploy dynamic electricity pricing strategies incentivizing consumers to perform demand side management by adjusting their loads to match the current state of the network, i.e., shifting their loads from the peak periods to off peak periods. There are several ways to perform demand side management, including integration of intermittent energy sources such as photovoltaic (PV) power or wind power at the residential level, demand shaping, etc. [3]. In this paper we focus on the former solution, or more specifically,

This work is sponsored in part by a grant from the National Science Foundation. integrating PV power generation with the Smart Grid for residential usage.

Although integrating residential-level renewable energy sources into the Smart Grid will prove useful in reducing the usage of fossil fuels, one critical problem needs to be addressed. There exists a mismatch between the peak PV power generation time (usually at noon) and the peak load power consumption time for the residential user (usually in the evening.) This timing skew results in cases where the generated PV power cannot be optimally utilized for peak power shaving.

One effective solution of the above-mentioned problems will be incorporating storage system into the PV assisted Smart Grid for residential users. The proposed residential-level storage system shall store power from the Smart Grid during off peak periods of each day and (or) from the PV system, and provide power during the peak periods of that day for peak power shaving and energy cost reduction (since electrical energy tends to be most expensive during these peak hours.) The design of energy pricing-aware control algorithm for the residential storage system, which controls the charging and discharging of the storage system, is hence an important task.

A realistic electricity pricing function consists of both an energy price component, which is a time of usage (TOU) dependent function indicating the unit energy price during each time period of the billing period (a day, or a month, etc.), and a demand price component, which is an additional charge due to the peak power consumption in the billing period. The latter component is added to the price of energy consumed in order to prevent a case whereby all customers utilize their PV power generation and energy storage systems and/or schedule their loads such that a very large amount of power is demanded from the Smart Grid during low-cost time slots, which can subsequently result in power delivery failure for all customers.

Moreover, the size of the storage system is limited due to the relatively high cost of electrical energy storage elements. Therefore, it is important for the controller to forecast the future PV power generation and load power consumption profiles so that it can perform optimization of the total cost. References [4][5][6] are representative of PV power generation and load power consumption profile predictions. However, these methods are general profile predicting methods, not specifically designed to help a residential-level storage controller. The controller may not perform optimal electrical energy cost reduction with such prediction methods.

In this paper we consider the case of a residential Smart Grid user equipped with local PV power generation and energy storage systems. We consider a realistic electricity price function comprised of both energy and demand prices. First, we present novel PV power generation and load power consumption profile predictors specifically designed for the residential-level Smart Grid controller. Furthermore, to perform 
effective storage system control utilizing such prediction results to minimize total electricity cost, we separate the proposed control algorithm into two tiers, one performed at each decision epoch of the billing period to globally "plan" the future discharging and charging schemes of the storage system, and another one performed locally as system operates to compensate the prediction errors. The first tier of the control algorithm can be effectively implemented by solving a convex optimization problem at each decision epoch, while the second tier has a time complexity of $\mathrm{O}(1)$. Experimental results demonstrate the accuracy of PV generation and load consumption predictions, as well as the effectiveness of the proposed residential-level storage control algorithm.

\section{System MODEL AND COST FUNCTIONS}

In this paper, we consider an individual residential Smart Grid user equipped with PV power generation and energy storage systems, as shown in Figure 1. The PV system and storage system are connected to the residential-level DC bus via DC-DC conversion circuitries. The AC bus, which is further connected to the Smart Grid, is connected via an AC/DC interface to the residential-level DC bus. The residential-level AC load is connected to the AC bus.

We adopt a slotted time model, i.e., all system constraints as well as decisions are provided for discrete time intervals of equal length. More specifically, each day is divided into $T$ time slots, each of duration $D$. We use $T=96$ and $D=15$ minutes.

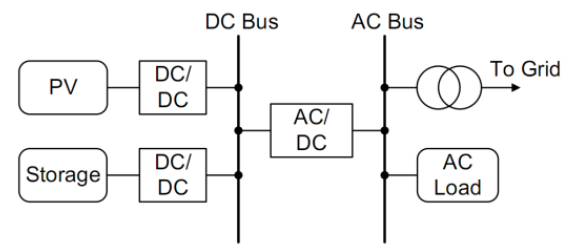

Figure 1. Block diagram showing the interface between PV array, storage system, residential load, and the Smart Grid.

We adopt a realistic electricity price function consisting of both the energy price component and the demand price component, with a billing period of a month [7]. Consider a specific day $i$ of a billing period. The residential load power consumption at the $j^{\text {th }}$ time slot of that day is denoted by $P_{\text {load }, i}[j]$. The output power values of PV and storage systems at the $j^{\text {th }}$ time slot are denoted by $P_{p v, i}[j]$ and $P_{s t, i}[j]$, respectively, in which $P_{s t, i}[j]$ can be positive (discharging from the storage), negative (charging the storage), or zero. Therefore the power required from the Smart Grid, i.e., the grid power, at the $j^{\text {th }}$ time slot of the $i^{\text {th }}$ day, denoted by $P_{\text {grid, } i}[j]$, satisfies

$P_{\text {grid }, i}[j]=P_{\text {load }, i}[j]-P_{p v, i}[j]-P_{s t, i}[j]$

where $P_{\text {grid }, i}[j]$ can be positive (if the Smart Grid provides power for the residential usage), negative (if the residential system sells power back into the Grid), or zero.

As specified in [7], the electricity price function is preannounced by the utility company just before the start of each billing period, and the price function will not change until the start of the next billing period. Reference [7] also specifies five different time periods of each day, denoted by the term price periods, with (potentially) different unit energy prices and demand prices. These price periods are: the $1^{\text {st }}$ off peak $(\mathrm{OP})$ period from 00:00 to 09:59, the $1^{\text {st }}$ low peak (LP) period from 10:00 to 12:59, the high peak (HP) period from 13:00 to 16:59, the $2^{\text {nd }}$ LP period from 17:00 to 19:59, and the $2^{\text {nd }}$ OP period from 20:00 to 23:59. We denote the $1^{\text {st }} \mathrm{OP}, 1^{\text {st }} \mathrm{LP}, \mathrm{HP}, 2^{\text {nd }} \mathrm{LP}$, and $2^{\text {nd }}$ OP price periods of a day as the $1^{\text {st }}, 2^{\text {nd }}, 3^{\text {rd }}, 4^{\text {th }}$, and $5^{\text {th }}$ price periods of that day. We use $j \in S_{k}$ to denote the statement that the $j^{\text {th }}$ time slot belongs to the $k^{\text {th }}$ price period. We use $t_{s, k}$ and $t_{e, k}$ to denote the start time and end time of the $k^{\text {th }}$ price period in each day, respectively. Obviously, we have $t_{s, 1}=$ 00: 00, $t_{e, 5}=23: 59$, and $t_{s, k}=t_{e, k-1}$ for $2 \leq k \leq 5$.

We use Price_E $E_{O P}$, Price_E $E_{L P}$, and Price_ $E_{H P}$ to denote the unit energy price in the OP period (the $1^{\text {st }}$ and $5^{\text {th }}$ price periods), the LP period (the $2^{\text {nd }}$ and $4^{\text {th }}$ price periods), and the HP period (the $3^{\text {rd }}$ price period) of each day, respectively, and use Price $D_{L P}$, Price_ $D_{H P}$, and Price_ $D_{\text {Overall }}$ to denote the monthly demand price for the peak power demands drawn from the Grid during the LP period, the HP period, and the overall peak power demand of a billing period (a month), respectively. Obviously we have Price_ $E_{H P}>$ Price_ $E_{L P}>$ Price $E_{O P}$, and Price $D_{H P}>$ Price_ $D_{L P}$. Hence, the cost we pay of a billing period due to the energy price component is given by

Cost $_{E}=$ Price $_{-} E_{O P} \cdot \sum_{i=1}^{30} \sum_{j \in S_{1} \cup S_{5}} P_{\text {grid }, i}[j] \cdot D+$
Price $_{-} E_{L P} \cdot \sum_{i=1}^{30} \sum_{j \in S_{2} \cup S_{4}} P_{\text {grid }, i}[j] D+$ Price $_{-} E_{H P} \cdot \sum_{i=1}^{30} \sum_{j \in S_{3}} P_{\text {grid }, i}[j] D$

and the cost we have to pay of a billing period due to the demand price component is given by

Cost $_{D}=$ Price $_{-} D_{L P} \cdot \max _{1 \leq i \leq 30, j \in S_{2} \cup S_{4}} P_{\text {grid }, i}[j]+$

Price_ $D_{H P} \max _{1 \leq i \leq 30, j \in S_{3}} P_{\text {grid }, i}[j]+$ Price_ $D_{\text {Overall }} \max _{1 \leq i \leq 30,1 \leq j \leq 96} P_{\text {grid }, i}[j]$

Obviously, the total cost for the residential user of a billing period is the sum of two aforesaid cost components.

\section{PV AND LOAD POWER PROFILE PREDICTION}

Accurate prediction of the PV power generation and load power consumption profiles is extremely important for the development of residential-level storage control algorithms. Due to the fact that predicting the complete load (or PV) power profile is difficult and unnecessary, we use predictors to forecast the peak and average load power consumption (or PV power generation) values for different price periods in each day, i.e., one predictor for the $1^{\text {st }}$ OP period, one for the $1^{\text {st }} \mathrm{LP}$ period, one for the HP period, one for the $2^{\text {nd }} \mathrm{LP}$ period, and one for the $2^{\text {nd }}$ OP period. Subsequently, we shall reconstruct the approximate load (or PV) power profile for each day based on the predicted average and peak values in each price period.

In the following we describe the peak power predictors; the average predictors can be realized in the same way. We present the peak load power consumption predictor in Section III-A and peak PV power generation predictor in Section III-B. The proposed load power consumption and PV power generation prediction algorithms consist of an initial prediction phase followed by an intra-day refinement phase, as explained below.

Consider the peak power (consumption or generation) prediction for the $i^{\text {th }}$ day of a billing period (i.e., a month.) The initial prediction of the peak power refers to prediction performed at time 00:00 $\left(t_{s, 1}\right)$ of the $i^{\text {th }}$ day, for the peak load power consumption (or PV power generation) in all five price periods of the $i^{\text {th }}$ day. The intra-day refinement of peak power prediction may be performed at the start time of the $k^{\text {th }}$ $(1<k \leq 5)$ price period, i.e., $t_{s, k}$, of the $i^{\text {th }}$ day, with the goal of refining the initial prediction of the peak power values in the $k^{\text {th }},(k+1)^{\mathrm{st}}, \ldots, 5^{\text {th }}$ price periods. Motivation for the intra-day refinement is that at time $t_{s, k}(1<k \leq 5)$, the actual peak load power consumption (or PV power generation) values in the $1^{\text {st }}$, $2^{\text {nd }}, \ldots,(k-1)^{\mathrm{st}}$ price periods of the $i^{\text {th }}$ day are known. This 
information can thus be used to improve the accuracy of peak power prediction for the remaining price periods in the day.

\section{A. Prediction Algorithm for Peak Load Power Consumption}

For the initial prediction phase of peak load power consumption, an adaptive regression-based algorithm is used. Consider that we are at time $t_{s, 1}=00: 00$ of the $i^{\text {th }}$ day. The peak load power values in the $k^{\text {th }}$ price period of the $i^{\text {th }}$ day $(1 \leq k \leq 5)$ are predicted as follows:

Predict $_{i, k}=\theta_{i, k}(0)+\sum_{l=1}^{n} \theta_{i, k}(l) \cdot$ Feature $_{i, k}(l)$

where $\boldsymbol{\theta}_{i, k}=\left(\theta_{i, k}(0), \theta_{i, k}(1), \ldots, \theta_{i, k}(n)\right)$ is a dynamicallyupdated coefficient vector with $n$ elements initialized to $1 / n$. In addition, the feature vector Feature $_{i, k}=$ (Feature $_{i, k}(1)$, Feature $_{i, k}(2), \ldots$, Feature $\left._{i, k}(n)\right)$ captures the actual values of peak load power consumption sampled at some points of interest. We have found that a value of $n=5$ with the feature vector defined as follows yields the best prediction results:

Feature $_{i, k}=\left(\right.$ Actual $_{i-1, k}$, Actual $_{i-2, k}$,

$$
\text { Actual } \left._{i-7, k}, \text { Actual }_{i-14, k}, \text { Actual }_{i-21, k}\right)
$$

where Actual $_{*, k}$ denote actual peak load power consumptions in the $k^{\text {th }}$ price period of the $(i-1)^{\text {st }}$ day, the $(i-2)^{\text {nd }}$ day, the $(i-7)^{\text {th }}$ day, the $(i-14)^{\mathrm{th}}$ day, and the $(i-21)^{\mathrm{st}}$ day, respectively.

Utilizing a stochastic gradient descent optimization method (see [8] for details), the vector $\boldsymbol{\theta}_{i, k}$ is updated as follows:

$\boldsymbol{\theta}_{i+1, k} \leftarrow \boldsymbol{\theta}_{i, k}+\alpha \cdot\left(\right.$ Actual $_{i, k}-$ Predict $\left._{i, k}\right) \cdot$ Feature $_{i, k}$

where $0<\alpha<1$ is a learning parameter.

For the intra-day refinement phase of peak load power consumption prediction, consider that we are currently at time instance $t_{s, k}$ of day $i$, and we intend to refine the initial prediction results of the peak load power consumption values in the $\hat{k}^{\text {th }}(k \leq \hat{k} \leq 5)$ price period of that day. We denote the result of refinement as Refine $_{i, \hat{k}}$. Since at that time $t_{s, k}$ the actual peak load power consumption in the $(k-1)^{\mathrm{st}}$ price period is known, we calculate Refine $_{i, \hat{k}}$ as follows:

Refine $_{i, \hat{k}} \leftarrow(1-\gamma) \cdot$ Predict $_{i, \hat{k}}+\gamma \cdot \frac{\text { Actual }_{i, k-1}}{\text { Predict }_{i, k-1}} \cdot$ Predict $_{i, \hat{k}}$

where $0<\gamma<1$ is another learning parameter.

The intuition for this update equation is that if the actual peak load power consumption in the $(k-1)^{\mathrm{st}}$ price period is higher than the predicted peak load power consumption in that period, i.e., Actual $_{i, k-1}>$ Predict $_{i, k-1}$, it is highly likely that the actual load power consumption in the $\hat{k}^{\text {th }}$ price period $(k \leq \hat{k} \leq 5)$ will also be higher than the predicted peak load power consumption in that period, and vice versa.

\section{B. Prediction Algorithm for Peak PV Power Generation}

For the peak PV power generation prediction, an important observation is that the actual peak PV power generation over a specific price period (e.g., the $k^{\text {th }}$ price period) in the $i^{\text {th }}$ day of a billing period may be viewed as the peak PV power generation over the $k^{\text {th }}$ price period for a sunny day, multiplied by a decay factor, representing the effect of clouds, if that day is cloudy. Obviously, such sunny day peak PV power generation over the $k^{\text {th }}$ period $(1 \leq k \leq 5)$ varies with the change in seasons, i.e., it is higher in the summer and lower in the winter. This effect is however captured by a smoothing operation as described below (cf. equations (9) and (10).) Therefore, for each day, we use the initial prediction, performed at the beginning of the day, mainly to predict the sunny day peak $P V$ power generation over each price period of that day of the billing period. Next, we rely on the intra-day refinement, performed at the start time of the $k^{\text {th }}$ $(1<k \leq 5)$ price period, i.e., $t_{s, k}$, of that day, to predict the decay factors (and subsequently, the actual peak PV power generations) in the remaining price periods.

In the initial prediction phase, we adopt a variant of the well-known exponential average based prediction method, for effectively predicting the sunny day peak PV power generation in each price period of day. Consider that we are at time $t_{s, 1}=00: 00$ of the $i^{\text {th }}$ day, and we want to derive the prediction value of the sunny day peak PV power generation in the $k^{\text {th }}(1 \leq k \leq 5)$ price period of that day, denoted by Predict $_{i, k}$, based on the prediction of sunny day peak PV power generation in the $k^{\text {th }}$ price period of the $(i-1)^{\text {st }}$ day, denoted by Predict $_{i-1, k}$, and the actual peak PV power generation in the $k^{\text {th }}$ price period of the $(i-1)^{\text {st }}$ day, denoted by Actual $_{i-1, k}$. Note that we must also capture and predict seasonal change of the sunny day peak PV power generation values, while filtering out random power decaying effects due to the presence of clouds. This is a smoothing operation. Thus the Predict $_{i, k}$ value can be calculated as follows:

Predict $_{i, k}=\beta\left(\right.$ Predict $_{i-1, k}$, Actual $\left._{i-1, k}\right) \cdot$ Actual $_{i-1, k}$

$+\left(1-\beta\left(\right.\right.$ Predict $_{i-1, k}$, Actual $\left.\left._{i-1, k}\right)\right) \cdot$ Predict $_{i-1, k}$

In the above equation, the learning rate function $\beta$ Predict $_{i-1, k}$, Actual $\left._{i-1, k}\right)$ is set to:

$\beta_{0}$, if Predict $_{i-1, k}<$ Actual $_{i-1, k}$

$\beta_{0} \cdot e^{\left.-\lambda \cdot \text { Predict }_{i-1, k}-\text { Actual }_{i-1, k}\right)}$, otherwise

where $\beta_{0}$ is basis learning rate, and $\lambda$ is a decaying parameter.

The motivation for this smoothing operation is as follows. Since (i) we want to predict the seasonal change of sunny day peak PV power generations while filtering out the effect of clouds and (ii) Actual $_{i-1, k} \ll$ Predict $_{i-1, k}$ only if there are clouds, it is natural that our new predicted sunny day peak PV power generation value Predict ${ }_{i, k}$ should not be so much influenced by the Actual $_{i-1, k}$ value (which is strongly affected by the clouds.) Therefore we adopt the exponentially decaying learning rate function (9), instead of the constant learning rate in the original exponential average based prediction algorithm.

The intra-day refinement phase of PV power generation prediction, which is performed at the start time of the $k^{\text {th }}(1<$ $k \leq 5)$ price period of each day to predict the decay factors (and subsequently, the actual peak PV power generations) in the remaining price periods, can be implemented via exactly the same algorithm (Eqn. (7)) as the intra-day refinement phase of load power consumption prediction.

\section{RESIDENTIAL-LEVEL STORAGE CONTROL}

In this section, we discuss the proposed residential-level storage control algorithm, which could effectively utilize the combination of PV power generation and load power consumption predictions to minimize the total electricity cost over each billing period. The proposed storage control algorithm is hierarchical in that it consists of a global control tier, which is performed at each decision epoch (to be precisely defined later) to "plan" the future discharging and charging schemes of the local energy storage for the remainder of the day, and a local control tier, which is performed locally (and much more frequently) at regular fixed-length timing intervals called time slots to mitigate the effect of prediction errors.

Consider that we are currently in the $i^{\text {th }}$ day of a billing period (a month.) Then the decision epochs in that day are defined to be the start times of each price period of that day (excluding the $1^{\text {st }}$ OP period), i.e., $t_{s, k}$ for $1<k \leq 5$. At each decision epoch, the storage controller gets information about 
the PV power generation and load power consumption profile characteristics (peak and average power) over the $k^{\text {th }},(k+1)^{\mathrm{st}}$, $\ldots, 5^{\text {th }}$ price periods from intra-day refinement phase of PV and load predictors performed just at that decision epoch $t_{s, k}$, and it is going to globally "plan" the discharging and charging scheme of the storage in the remaining price periods of the day. Obviously the "globally planned storage charging/discharging scheme" on the $(k+1)^{\text {st }}, \ldots, 5^{\text {th }}$ price periods obtained at decision epoch $t_{s, k}$ may be modified in the global planning process at the following decision epoch $t_{s, k+1}$. Note that the start time of the $1^{\text {st }}$ OP period is not considered as decision epoch. This is because it is very unlikely to have a peak power demand to be drawn from the Grid during that price period. Therefore during the $1^{\text {st }}$ OP period, the storage system is being charged, instead of being discharged as in the following $1^{\text {st }} \mathrm{LP}$, the HP, and the $2^{\text {nd }}$ LP periods. Hence we simply assume that peak power demand to be drawn from the Grid, which may affect the monthly demand price, will not occur during the $1^{\text {st }}$ OP period, and furthermore, at the end of that price period (at time $t_{s, 2}$ ), the storage system will be (nearly) fully charged.

We use $E_{i, k}$ to denote the available storage energy at decision epoch $t_{s, k}$ of the $i^{\text {th }}$ day, and we have $E_{i, 2} \approx E_{\text {full }}$ in which $E_{f u l l}$ is the storage energy when fully charged, and $E_{i, 2} \geq E_{i, 3} \geq E_{i, 4} \geq E_{i, 5}$, since storage is being discharged in the $1^{\text {st }} \mathrm{LP}$, the HP, and the $2^{\text {nd }}$ LP period.

\section{A. The Global Control Tier}

In contrast to the other parts of the paper, in the global control tier of the storage control algorithm, we use a continuous-time based system model. Consider that we are currently at decision epoch $t_{s, k_{0}}\left(1<k_{0} \leq 5\right)$ of the $i^{\text {th }}$ day. In this algorithm we use the predicted net load power consumption profile of the remaining of the $i^{\text {th }}$ day, denoted by $P_{n e t, i}(t), t \in\left[t_{s, k_{0}}, t_{e, 5}=24\right]$, which equals to the predicted load power consumption profile minus the predicted PV power generation profile. Such predicted $P_{n e t, i}(t)$ profile can be reconstructed from the intra-day refinement phase of PV and load peak and average power predictions, as shall be discussed later. The storage output power, which is the control variable of the global tier of the storage control algorithm, is denoted by $P_{s t, i}(t)$. Therefore the (predicted) power drawn from the Grid (the grid power), denoted by $P_{\text {grid,i }}(t)$, can be calculated via $P_{\text {grid }, i}(t)=P_{\text {net }, i}(t)-P_{\text {st }, i}(t)$.

Consider a specific price period (the $k^{\text {th }}$ price period with $1<k \leq 5$, for instance) of the $i^{\text {th }}$ day, as shown in Figure 2 . We use $p_{k}$ and $a_{k}$ to denote the predicted peak and average net load power consumption values in that price period, respectively. Furthermore, we use $p_{\text {load }, k}$ and $a_{\text {load, } k}$ to denote the predicted peak and average load power consumption values, and use $p_{p v, k}$ and $a_{p v, k}$ to denote the predicted peak and average PV power generation values, over the $k^{\text {th }}$ price period of the $i^{\text {th }}$ day, respectively. Note that the index $i$ of such $p_{k}, a_{k}$, etc. values has been dropped for the conciseness in notation. The above-defined $p_{\text {load }, k}, a_{\text {load }, k}, p_{p v, k}$, and $a_{p v, k}$ values can be obtained from the intra-day refinement of PV and load predictions, and the $p_{k}$ and $a_{k}$ values can be approximately calculated in the following way:

$p_{k}=p_{l o a d, k}-\left(2 a_{p v, k}-p_{p v, k}\right)$

$a_{k}=a_{l o a d, k}-a_{p v, k}$

Then the net load power consumption during the $k^{\text {th }}(1<k \leq$ $5)$ price period of the $i^{\text {th }}$ day, i.e., $P_{n e t, i}(t), t \in\left[t_{s, k}, t_{e, k}\right]$, is assumed to be uniformly distributed between the lowest value $2 a_{k}-p_{k}$ and the highest value $p_{k}$. We draw such predicted net load power consumption curve as line segment (a) in Figure 2.

The role of storage discharging in the price period of interest is to make the power drawn from the Grid $P_{\text {grid }, i}(t)$ lower than the net load power $P_{n e t, i}(t)$. It can be proved that when the total predicted energy drawn from the storage system during the $k^{\text {th }}$ price period of the $i^{\text {th }}$ day is fixed, then the optimal (predicted) grid power profile in terms of grid power peak minimization is given by $\min \left(P_{\text {net }, i}(t), P_{\text {target }, k}\right)$ for $t \in\left[t_{s, k}, t_{e, k}\right]$, in which the $P_{\text {target }, k}$ value can be determined by the aforesaid total predicted storage energy drawn in such price period. The optimal (predicted) grid power profile in the $k^{\text {th }}$ price period of the $i_{k \text { th }}^{\text {th }}$ Price Period is shown as curve (b) in Figure 2.

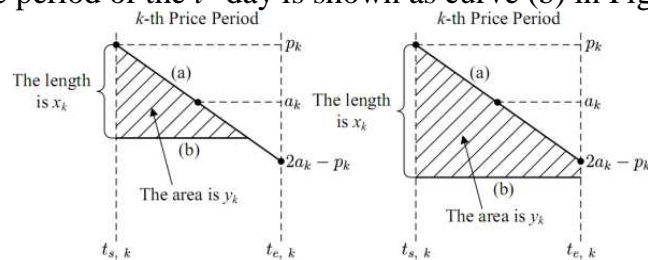

Figure 2. Relationship between the predicted net load power, storage output power and grid power profiles in the $k^{\text {th }}$ price period of the $i^{\text {th }}$ day.

We use $x_{k}$ to denote the maximum power reduction between the predicted net load power profile and the predicted grid power profile in the $k^{\text {th }}$ price period of the $i^{\text {th }}$ day, i.e., $x_{k}=p_{k}-P_{\text {target }, k}$, as shown in Figure 2 . We use $y_{k}$ to denote the total predicted energy drawn from storage during such price period. Obviously, $y_{k}$ is a function of $x_{k}$, denoted by $y_{k}=f_{k}\left(x_{k}\right)$. In fact, $y_{k}$ is a convex and monotonically increasing function of $x_{k}$, because: (i) $d f_{k}\left(x_{k}\right) / d x_{k}>0$, and (ii) $d f_{k}\left(x_{k}\right) / d x_{k}$ is the smallest at the beginning $\left(x_{k}=0\right)$, and then gradually increases as $x_{k}$ becomes larger.

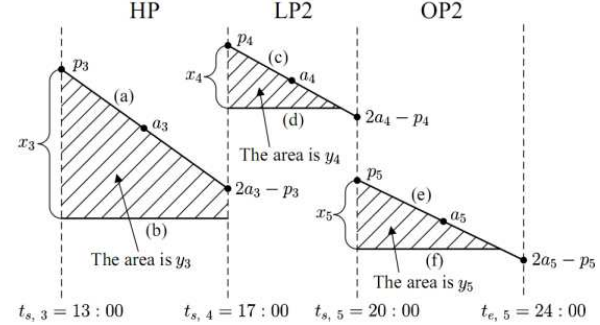

Figure 3. The optimal control problem at decision epoch $t_{s, 3}=13: 00$.

Now we return to the optimal storage control problem at decision epoch $t_{s, k_{0}}\left(1<k_{0} \leq 5\right)$ of the $i^{\text {th }}$ day, as illustrated in Figure $3\left(k_{0}=3\right.$ in this case.) At that time, the predicted $p_{k}$ and $a_{k}$ values for $k_{0} \leq k \leq 5$ can be derived from the intraday refinement of PV power generation and load power consumption predictions at decision epoch $t_{s, k_{0}}$, as well as Eqn. (10), and the storage energy at decision epoch $t_{s, k_{0}}$ is given by $E_{i, k_{0}}$. Furthermore, we denote the peak grid power (actual peak power) consumptions observed so far over the OP, LP, and HP price periods in this billing period of interest by $P e a k_{O P}$, $P e a k_{L P}$, and Peak ${ }_{H P}$, respectively. Obviously, such Peak $k_{O P}$, $P e a k_{L P}$, and $P e a k_{H P}$ values are initialized to be zero at the beginning of billing period. The $x_{k}$ values for $k_{0} \leq k \leq 5$ in this optimal storage control problem are variables, and we have $y_{k}=f_{k}\left(x_{k}\right)$ for $k_{0} \leq k \leq 5$. The objective of the optimal storage control problem is to find the optimal $x_{k}$ values for $k_{0} \leq k \leq 5$, subject to storage energy constraint $\sum_{k=k_{0}}^{5} y_{k} \leq$ 
$E_{i, k_{0}}$, for helping minimize the total cost over the billing period of interest, with total cost function given in (2), (3).

Then the proposed (near-) optimal storage control algorithm at decision epoch $t_{s, k_{0}}$ is given by the following. First we check whether storage has enough energy for peak shaving such that the (predicted) cost due to demand price in the billing period will not increase in the $i^{\text {th }}$ day, i.e., the (predicted) grid power $P_{\text {net }, i}(t)$ in the LP, HP, and OP periods in the remaining of the $i^{\text {th }}$ day does not exceed $P e a k_{L P}, P e a k_{H P}$, and $\max \left(P_{e} a k_{O P}\right.$, $\left.\operatorname{Peak}_{L P}, P e a k_{H P}\right)$, respectively. More specifically, we set:

$x_{2} \leftarrow \max \left(0, p_{2}-P e a k_{L P}\right)$, if $k_{0}=2$

$x_{3} \leftarrow \max \left(0, p_{3}-P e a k_{H P}\right)$, if $k_{0} \leq 3$

$x_{4} \leftarrow \max \left(0, p_{4}-P e a k_{L P}\right)$, if $k_{0} \leq 4$

$x_{5} \leftarrow \max \left(0, p_{5}-\max \left(\right.\right.$ Peak $_{O P}$, Peak $_{L P}$, Peak $\left.\left.k_{H P}\right)\right)$

Then we compare between $E_{i, k_{0}}$ and $\sum_{k=k_{0}}^{5} y_{k}=\sum_{k=k_{0}}^{5} f_{k}\left(x_{k}\right)$, and have the following two cases based on comparison results.

Case I $\left(E_{i, k_{0}} \geq \sum_{k=k_{0}}^{5} f_{k}\left(x_{k}\right)\right)$ : In this case the storage energy is adequate for peak shaving such that the (predicted) cost due to demand price in the billing period will not increase in the remaining of the $i^{\text {th }}$ day. In this case we further minimize the (predicted) cost due to unit energy price in the remaining of the $i^{\text {th }}$ day, subject to the constraint that the (predicted) cost due to demand price will not increase in that day. We call such problem cost minimization with adequate energy (CMAE), with deterministic solution as follows:

(1) If $k_{0}=2$, we set $x_{3} \leftarrow f_{3}^{-1}\left(E_{i, 2}-f_{2}\left(x_{2}\right)-f_{4}\left(x_{4}\right)-\right.$ $\left.f_{5}\left(x_{5}\right)\right)$, and then $x_{3}$ will become larger than its original value $\max \left(0, p_{3}-\right.$ Peak $\left.k_{H P}\right)$. We keep the $x_{2}, x_{4}$, and $x_{5}$ values the same as before. This is because the unit energy price in the HP period is the highest among each day, and thus we are going to use all the (predicted) surplus storage energy in the HP period for total cost minimization.

(2) If $k_{0}=3$, we set $x_{3} \leftarrow f_{3}^{-1}\left(E_{i, 3}-f_{4}\left(x_{4}\right)-f_{5}\left(x_{5}\right)\right)$, and keep the $x_{4}$ and $x_{5}$ values unchanged.

(3) If $k_{0}=4$, we set $x_{4} \leftarrow f_{4}^{-1}\left(E_{i, 4}-f_{5}\left(x_{5}\right)\right)$, and keep the $x_{5}$ value the same as before.

(4) If $k_{0}=5$, we simply leave the $x_{5}$ value as it was. This is because the unit energy price in the $2^{\text {nd }}$ OP price period is the lowest among each day.

Case II $\left(E_{i, k_{0}}<\sum_{k=k_{0}}^{5} f_{k}\left(x_{k}\right)\right)$ : In this case the storage energy is not adequate for peak shaving and hence we have to make the predicted peak grid power consumption over at least one of the LP, HP and OP price periods of the $i^{\text {th }}$ day exceed the Peak $_{L P}, P e a k_{H P}$, and $\max \left(\right.$ Peak $\left._{O P}, P e a k_{L P}, P e a k_{H P}\right)$ values, respectively. We are going to solve the following optimization problem, called the peak shaving with inadequate energy (PSIE) problem, such that the (predicted) cost increase due to demand price in the $i^{\text {th }}$ day will be minimized.

\section{The PSIE Optimization Problem}

Find the optimal values $x_{k}$ for $k_{0} \leq k \leq 5$.

\section{Minimize}

Price_ $D_{L P} \cdot \max \left\{\right.$ Peak $\left._{L P}, \mathbf{I}\left[k_{0}=2\right]\left(p_{2}-x_{2}\right), \mathbf{I}\left[k_{0} \leq 4\right]\left(p_{4}-x_{4}\right)\right\}+$

Price_D $D_{H P} \cdot \max \left\{\right.$ Peak $\left._{H P}, \mathbf{I}\left[k_{0} \leq 3\right] \cdot\left(p_{3}-x_{3}\right)\right\}+$

Price_D $_{\text {Overall }} \cdot \max \left\{\right.$ Peak $_{L P}$, Peak $k_{H P}$, Peak $k_{O P}$,

$\left.\mathbf{I}\left[k_{0}=2\right]\left(p_{2}-x_{2}\right), \mathbf{I}\left[k_{0} \leq 3\right]\left(p_{3}-x_{3}\right), \mathbf{I}\left[k_{0} \leq 4\right]\left(p_{4}-x_{4}\right), p_{5}-x_{5}\right\}$

in which $\mathbf{I}[x]$ is the indicator function, which equals to one if statement $x$ is true, and equals to zero otherwise.

Subject to:

$x_{k} \geq 0$, for $k_{0} \leq k \leq 5$ $\sum_{k=k_{0}}^{5} f_{k}\left(x_{k}\right) \leq E_{i, k_{0}}$

Remember that the function $f_{k}\left(x_{k}\right)$ is a convex and monotonically increasing function over $x_{k}$ for $k_{0} \leq k \leq 5$, the above PSIE problem is a convex optimization problem since it has convex objective function and convex inequality constraints, and therefore it can be solved in polynomial time, using convex optimization techniques such as [9].

\section{B. The Local Control Tier}

The local control tier of storage control algorithm shall be performed with system operates to compensate prediction errors. In this part we return to the slotted time model described in Section II. Consider that we are currently at the $j^{\text {th }}$ time slot, which belongs to the $k^{\text {th }}$ price period $(1<k \leq 5)$, of the $i^{\text {th }}$ day of a billing period. At that time we have the $P_{\text {target }, k}$ value which equals to $p_{k}-x_{k}$ of the $k^{\text {th }}$ price period, derived from the global tier of storage control algorithm performed at decision epoch $t_{s, k}$. Besides, we also have the actual net load power consumption value $P_{n e t, i}[j]=P_{l o a d, i}[j]-P_{p v, i}[j]$. Generally speaking, the basic job of the local tier of storage control algorithm is to seek to make the grid power $P_{\text {grid }, i}[j]=$ $P_{n e t, i}[j]-P_{s t, i}[j]$ no more than the $P_{\text {target }, k}$ value through controlling the storage output power $P_{s t, i}[j]$. Moreover, such algorithm should also make sure that the physical limitations of the storage system are not violated, i.e., the stored energy in the storage system will not exceed the maximum value $E_{f u l l}$, or become less than zero, at the end of the $j^{\text {th }}$ time slot. The proposed local tier of storage control algorithm is given by:

Local tier of storage controller at time slot $j, \operatorname{price} \operatorname{period} k$, day $i$ Assume that at the beginning of the $j$-th time slot, the stored energy in the storage system is $E_{i}[j]$.

If $P_{\text {net }, i}[j]>P_{\text {target }, k}$

$$
P_{s t, i}[j] \leftarrow \min \left(P_{\text {net }, i}[j]-P_{\text {target }, k}, \frac{E_{i}[j]}{D}\right) .
$$

Else If we are currently at the $2^{\text {nd }} \mathrm{OP}$ period, i.e., $k=5$

$P_{s t, i}[j] \leftarrow \max \left(P_{n e t, i}[j]-P_{\text {target }, k},-\frac{E_{\text {full }}-E_{i}[j]}{D}\right)$.

Else $P_{s t, i}[j] \leftarrow 0$.

Set $P_{\text {target }, k} \leftarrow \max \left(P_{\text {target }, k}, P_{\text {net }, i}[j]-P_{s t, i}[j]\right)$.

\section{EXPERIMENTAL RESULTS}

In this section we present the experimental results on load power consumption and PV power generation prediction algorithms proposed for residential-level Smart Grid users, as well as on the effectiveness of the proposed residential-level storage control algorithm. The PV power profiles used in our experiments are measured at Duffield, VA, in the year 2007, while the electric load data come from the Baltimore Gas and Electric Company, also measured in the year 2007.

\section{A. Load and PV Power Profile Predictions}

In this section we show some representative experimental results on the accuracy of the peak load power consumption and PV power generation predictions. The average load power consumption and PV power generation prediction results are similar, and are not shown in this paper due to space limitation. 


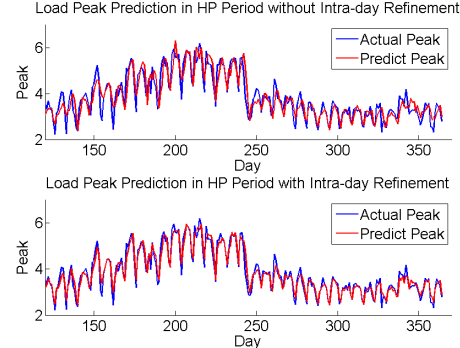

Figure 4. Comparison between the peak load power consumption prediction results from initial prediction (top) and from intra-day refinement at time

$t_{s, 3}=13: 00$ (bottom) and actual peak load power consumption results.

Figure 4 compares between the peak load power consumption prediction results and the actual peak load power consumption results in the HP period of each day in a year. The peak load power consumption prediction results shown in the top subfigure of Figure 4 come from the initial prediction performed at time 00:00 of each day, while the prediction results shown in the bottom subfigure come from the intra-day refinement performed at time $t_{s, 3}=13: 00$. Data in the first 120 days of the year are used for initial training, and thus the peak power consumption prediction results over those days are not shown in Figure 4. It can be observed from Figure 4 that our proposed adaptive regression-based initial prediction algorithm is effective in load power consumption prediction, resulting in an average prediction error of about $8 \%$. The average prediction error can be further reduced to less than $4 \%$, i.e., less than $50 \%$ of the average prediction error in initial prediction, by the use of intra-day refinement.

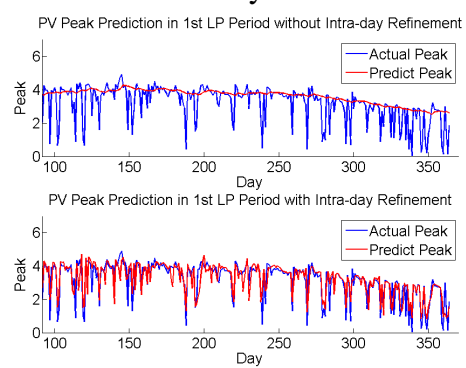

Figure 5. Comparison between the peak PV power generation prediction results from the initial prediction (top) and from intra-day refinement at time

$t_{s, 2}=10: 00$ (bottom) and actual peak PV power generation results.

Figure 5 compares between the peak PV power generation prediction results with the actual PV power generation results in the $1^{\text {st }}$ LP period of each day in a year. The peak PV power generation prediction results shown in the top subfigure of Figure 5 come from the initial prediction performed at time 00: 00 of each day, while the prediction results shown in the bottom subfigure come from intra-day refinement performed at $t_{s, 2}=10: 00$. Data in the first 90 days are used for initial training, and thus the peak PV power generation prediction results over those days are not shown. It can be observed that our modified exponential average-based initial prediction algorithm is effective in predicting the sunny day peak PV power generation over each day in a year. The proposed intraday refinement technique also proves itself effective in predicting the decay factors due to clouds.

\section{B. Residential-Level Storage Control Algorithm}

In our experiments, the residential Smart Grid user is equipped with the load devices and PV system with power consumption and generation profiles same as the profiles used in Section V-A, as well as a storage system which could perform peak shaving and energy cost reduction. We define the cost saving capability of a storage control algorithm to be the average monthly cost saving due to the additional storage system, compared to the same residential Smart Grid user equipped only with the PV system. We compare the cost saving capabilities of our proposed storage control algorithm, with two baseline algorithms. The first baseline algorithm is a relatively simple algorithm which charges the storage system from the Grid during the OP period with constant power, and distributes all the available energy stored in the storage system evenly in the HP and LP periods. The second baseline algorithm is a relatively advanced algorithm which, although still charges the storage system from the Grid during the OP period with constant power, distributes its available energy with constant storage output power $P_{s t, H P}^{B a s e 2}$ in the HP period and with constant storage output power $P_{S t, L P}^{B a s e 2}$ in the LP period. Moreover, the $P_{s t, H P}^{B a s e 2}$ and $P_{s t, L P}^{B a s e 2}$ values satisfy:

$P_{s t, H P}^{B a s e 2} / P_{s t, L P}^{B a s e 2}=$ Price_ $D_{H P} /$ Price_ $D_{L P}$

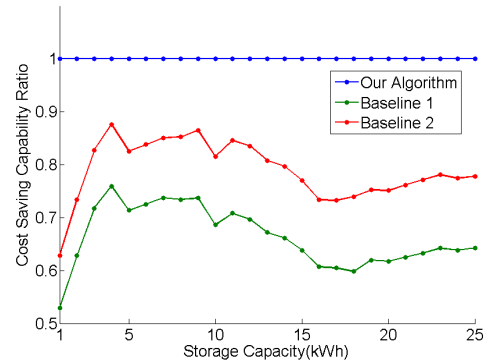

Figure 6. Comparison of the cost saving capabilities between proposed nearoptimal residential-level storage control algorithm, and baseline algorithms.

Figure 6 shows the comparison results on the cost saving capabilities between our proposed storage control algorithm and the baseline algorithms. The $\mathrm{x}$-axis of this figure is the total storage capacity, and the y-axis is the ratio of the cost saving capability of a storage control algorithm to the cost saving capability of our proposed algorithm. We can see that our proposed near-optimal storage control algorithm consistently outperforms the two baselines, with an average cost saving capability improvement of $51.93 \%$ than the first baseline algorithm and $27.25 \%$ than the second baseline algorithm.

\section{CONCLUSION}

This paper addresses the problem on integrating residentiallevel PV and storage systems into the smart grid for simultaneous peak shaving and total electricity cost minimization, making use of the dynamic energy pricing models. We first propose novel PV power generation and load power consumption profile forecasting techniques, specifically developed for the residential storage controller for performing peak shaving. We further propose the effective residential storage control algorithm, which consists of a global control tier performing at each decision epoch of a billing period to globally "plan" the future discharging/charging schemes of the storage system, and a local control tier performing along with system operation to compensate for the prediction errors.

\section{REFERENCES}

[1] L. D. Kannberg et al., "GridWise Energy System," PNNL-14396, Pacific Northwest National Laboratory, Richland, Sep. 2003.

[2] S. Kishore, L. V. Snyder, "Control Mechanisms for Residential Electricity Demand in SmartGrids," Proc. of Smart Grid Communications (SmartGridComm) Conference, 2010. 
[3] S. Caron and G. Kesidis, "Incentive-based Energy Consumption Scheduling Algorithms for the Smart Grid," Proc. of Smart Grid Communications (SmartGridComm) Conference, 2010.

[4] T. Hiyama and K. Kitabayashi, "Neural network based estimation of maximum power generation from PV module using environmental information," IEEE T. on Energy Conversion, 1997.

[5] C. Chen, B. Das, and D. J. Cook, "Energy prediction based on resident's activity," SensorKDD'10, 2010.
[6] L. Wei and Z-H. Han, "Short-term power load forecasting using improved ant colony clustering," WKDD, 2008.

[7] http://www.ladwp.com/ladwp/cms/ladwp001752.jsp

[8] C. M. Bishop, Pattern Recognition and Machine Learning, Springer, August 2006.

[9] M. Grant and S. Boyd, "CVX: Matlab software for disciplined convex programming, version 1.21.” http://cvxr.com/cvx, Feb. 2011 